CUPhysics $/ 5 / 2008$

\title{
Realistic searches on stretched exponential networks
}

\author{
Parongama Sen \\ Department of Physics, University of Calcutta, \\ 92 Acharya Prafulla Chandra Road, \\ Calcutta 700009, India.
}

\begin{abstract}
We consider navigation or search schemes on networks which have a degree distribution of the form $P(k) \propto \exp \left(-k^{\gamma}\right)$. In addition, the linking probability is taken to be dependent on social distances and is governed by a parameter $\lambda$. The searches are realistic in the sense that not all search chains can be completed. An estimate of $\mu=\rho / s_{d}$, where $\rho$ is the success rate and $s_{d}$ the dynamic path length, shows that for a network of $N$ nodes, $\mu \propto N^{-\delta}$ in general. Dynamic small world effect, i.e., $\delta \simeq 0$ is shown to exist in a restricted region of the $\lambda-\gamma$ plane.

PACS numbers: 89.75.Hc, 89.70.+c, 89.75.Fb
\end{abstract}

The subject of networks has emerged as a multidisciplinary field in which there has been tremendous activity in recent times [1, 2]. The interest in networks has grown due to the fact that although networks can be of diverse nature, there are some striking universal properties in their underlying structure. The most important property that appeared to be commonly occurring in networks is the small world property. This means that if any two nodes in the network is separated by an average number of $s$ steps, then $s \propto \ln (N)$, where $N$ is the total number of nodes in the network. In some networks, even slower variation (i.e., sub-logarithmic scaling) has been observed [3].

The first indication that networks have small world behaviour emerged from an experimental study by Milgram et al 4], in which it was shown that any two persons (in the USA) can be connected by an average number of six steps. Following the tremendous interest in the study of networks, new experiments have been done to verify this property in real social networks [5, 6]. Some studies which involve simulations on real networks [7, 8, 9] have been made also. Parallely, the question of navigation on small world networks has been addressed theoretically in many model networks [10, 11, 12, 13, 14, 15, 16, 17, 18, 19, 20, 21].

It must be noted that it is not necessary that a navigation or searching on a small world network would show the small world property, i.e., the dynamic paths $s_{d}$ may not scale as $\ln (N)$. This is because searching is done using local information only while the average shortest distances are calculated using the global knowledge of the network. This was explicitly shown by Kleinberg [10] in a theoretical study where nodes were placed on a two dimensional Euclidean space. Each node here has connections to its nearest neighbours as well as to neighbours at a distance $l$ with probability $P(l) \propto l^{-\alpha}$. Although the network is globally a small world for a range of values of $\alpha$, navigation on such networks using greedy algorithm showed a small world behaviour only at $\alpha=2$.

Conventionally a network is said to be searchable if there exist short dynamic paths scaling as $\ln (N)$. However, search chains have been shown to terminate unsuc- cessfully in many real experiments. In theoretical studies, this has been considered recently and it has been shown that with the possibility of failures, the scaling of the path lengths alone is not always a good measure of searchability [19]. The ratio of the success rate to the path lengths, on the other hand, gives a reliable measure. Based on this measure, the searchability on scale free networks and Euclidean networks have been analysed recently [19, 20]. The searchability depends on both the network structure and to a large extent on the searching strategy.

In the present work, we have considered a network with a degree distribution which is not scale-free but given by $P(k) \propto \exp \left(-k^{\gamma}\right)$, i. e., a stretched exponential behaviour. Here, we have a parameter $\gamma$ associated with the degree distribution; a large $\gamma$ value indicates a very fast decay of $P(k)$ such that the probability of having high degree nodes is very small. One the other hand, if $\gamma$ is small, such a probability may not be negligible even if the degree distribution is not scale-free. Moreover, we consider a characteristic feature attached to each node. The linking probability is taken to be dependent on the social distance or the difference of this characteristic feature between nodes. Thus, this is an attempt to construct a simple model of social network. A similar study was made in [19] where a scale-free degree distribution was used. However, in most social networks, one has a degree distribution with a faster than algebraic decay and therefore we have considered a stretched exponential degree distribution here.

The network is generated by a method described in detail in [19]. In brief, we first assign the degrees to the nodes according to the degree distribution. The characteristic feature called the similarity factor is measured by a variable $\xi$ varying from 0 to 1 and is assigned to each node randomly. The edges are then introduced between pairs of nodes $i$ and $j$ with the probability

$$
\mathcal{P}_{i, j} \propto\left|\xi_{i}-\xi_{j}\right|^{-\lambda},
$$

with $\lambda>0$. 

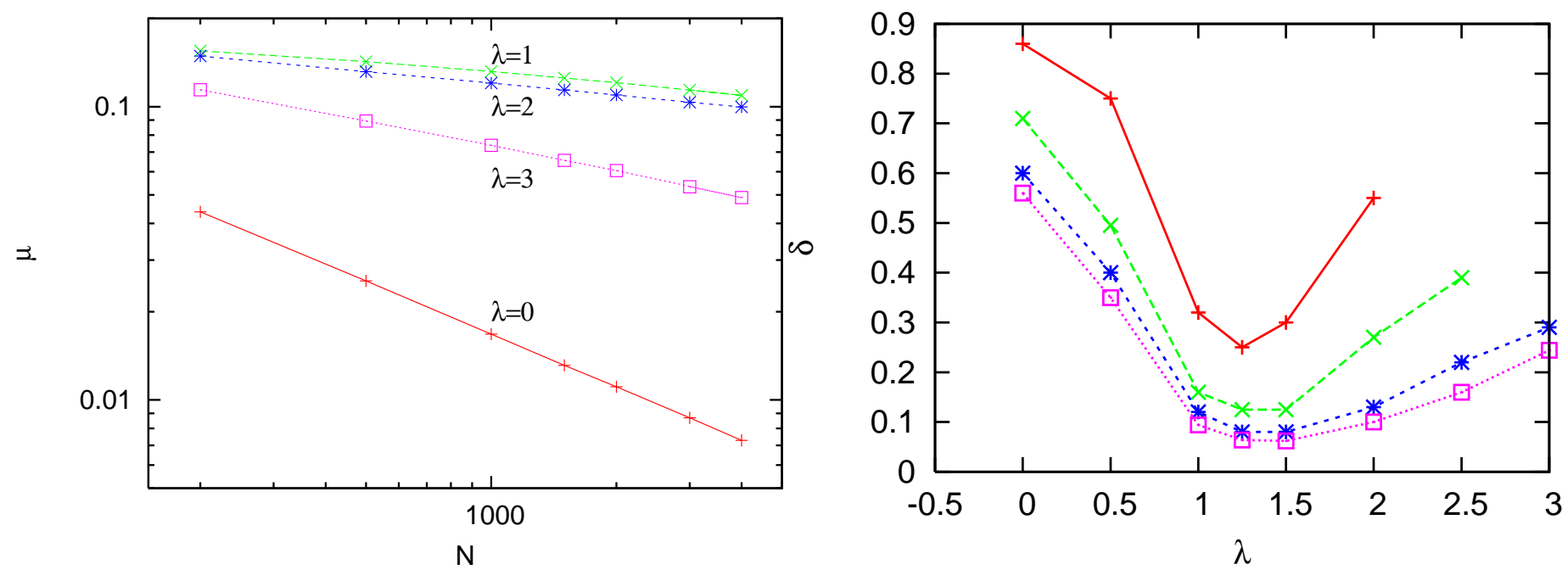

FIG. 1: The typical variations of the ratio $\mu$ of the success rate to the dynamic path lengths as a function of $N$ are shown for different values of $\lambda$ for $\gamma=0.4$. The slopes give the estimate of $\delta$.

Thus we will have a network in which similar nodes will try to link up for $\lambda>0$. A large value of $\lambda$ indicates that nodes which are very similar tend to link up while for $\lambda=0$, there are no correlations among nodes. The minimum and maximum allowed degrees are two and $N^{1 / 2}$ respectively.

We use an algorithm in which each node knows the similarity factor of its immediate neighbours as well as that of the target node. Nodes send messages to a neighbour most similar to the target node. Each node can receive the message only once. In case there is no node to which the message can be forwarded, the search will terminate. Conducting the searches between arbitrary source-target pairs, we estimate the success rates $\rho$ and the average dynamic paths $s_{d}$. The ratio $\mu=\rho / s_{d}$ is then studied as a function of the network size $N$.

We find that as in [19, 20], $\mu$ has a power law decay with $N$ with an exponent $\delta$ for all $\gamma$ and $\lambda$ in general except for very large values of $\lambda$ where there seems to be a correction to the power law scaling. $\delta$ lies between zero and one, as found in the previous studies. A smaller value of $\delta$ indicates a better searchability and in particular $\delta \simeq$ 0 would correspond to a dynamic small world (DSW) effect [19]. For each $\gamma$, we estimate $\delta$ as a function of $\lambda$. We find that for small $\gamma$, there is indeed a range of values of $\lambda$ where $\delta$ is very small, close to zero. On the other hand, for $\gamma>\gamma_{c}$, where $\gamma_{c}$ is close to 0.4 , there is no such region. In the present study, if $\delta<0.1$ (which means the decay could as well be logarithmic), we assume that there is a dynamic small world effect.

Fig. 1 shows typical variations of $\mu$ versus $N$ for $\gamma=0.4$ for various values of $\lambda$. The slope of these curves gives the exponent $\delta$. In Fig. 2, $\delta$ against $\lambda$ values are shown for various $\gamma$ where we observe that for low values of $\gamma$, there is a finite region where $\delta$ is less than 0.1 . This region
FIG. 2: The values of $\delta$ against $\lambda$ show that for low values of $\gamma$, a dynamic small world effect can be achieved for a finite interval of $\lambda$ values. The curves are shown for $\gamma=0.6, \gamma=$ $0.5, \gamma=0.4$ and $\gamma=0.3$, from top to bottom.

shrinks as $\gamma$ is increased. However, even when there is no dynamic small world effect, we find that $\delta$ reaches a minimum at $\lambda=\lambda_{\min }$ where $\lambda_{\min } \simeq 1.25$ independent of $\gamma$. Hence for a stretched exponential network in general, where the linking probability is dependent on the social distances parametrically as in (1), the network is most searchable for a fixed value of the parameter.

We have schematically shown the DSW region in the $\lambda-\gamma$ plane in Fig. 3. We would like to mention here that for the social network considered earlier, with a power law degree distribution $\left(P(k) \propto k^{-2}\right)$, DSW had been obtained for a finite range of value of $\lambda$ as well [19].

Thus we conclude that for realistic searches on social networks, where the degree distribution is given by a stretched exponential function, one can achieve dynamic small world effect as long as the degree distribution does not decay very fast and the network is neither too correlated nor too random as far as the similarity of nodes is concerned. In general, we find that while the quality of the searchability is determined by $\lambda, \gamma$ makes it quantitatively different, e.g., at smaller values of $\gamma$ where there is a larger number of highly connected nodes, the searchability increases.

We also find that in the DSW region, the success rate is almost a constant as a function of $N$, and the dynamic path lengths increase very slowly indicating a logarithmic increase. The absolute value of path lengths is also "small", e.g., for a network of size 4000, the dynamic path length is about 5 in the DSW region. This could explain the observed results of searching in real networks. Of course, in a real social search, a much more complicated dynamics is involved, with nodes having more than one characteristic feature and each node using its own searching strategy. 


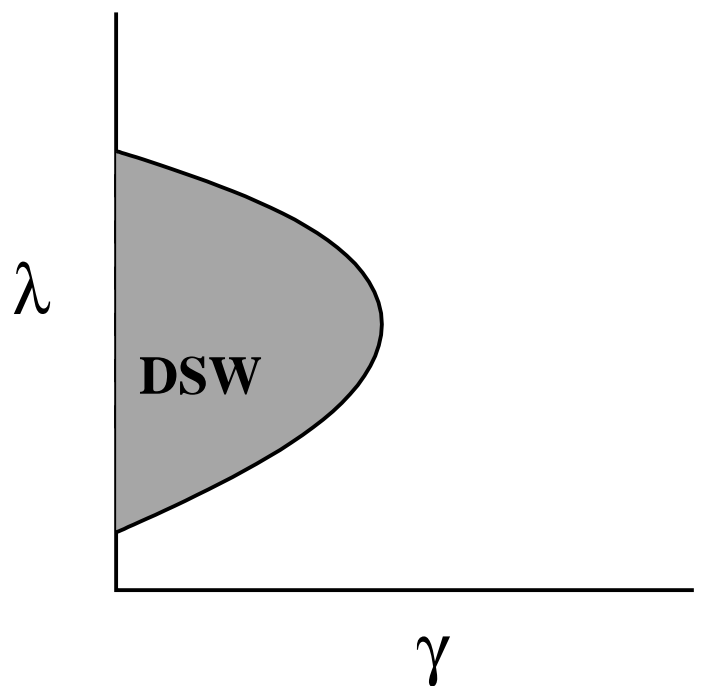

FIG. 3: Schematic picture of the $\lambda-\gamma$ plane showing the region with the dynamic small world effect.
Acknowledgement: Financial support from CSIR grant no. 3(1029)/05-EMR-II is acknowledged.
[1] D. J. Watts and S. H. Strogatz, Nature 393, 440 (1998); D. J. Watts, Small Worlds, Princeton Univ. Press, Princeton (1999).

[2] R. Albert and A.-L. Barabási, Rev. Mod. Phys. 74, 47 (2002).

[3] M. E. J. Newman, SIAM Rev. 45, 167 (2003).

[4] S. Milgram, Psychology Today 1, 60 (1967); J. Travers and S. Milgram, Sociometry 32, 425 (1969).

[5] P. D. Killworth and H. R. Bernard, Social Networks 1159 (1978).

[6] P. S. Dodds, R. Muhamad and D. J. Watts, Science 301, 827 (2003).

[7] L. A. Adamic and E. Adar, Social Networks 27, 187 (2005).

[8] I. Clarke, S. G. Miller, T. W. Hong, O. Sandberg and B. Wiley, IEEE Internet Computing 6, 40 (2002).

[9] D. Liben_Nowell, J. Novak, R. Kumar, P.Raghavan and A. Tomkins, PNAS 102, 11623 (2005).

[10] J. Kleinberg, Nature 406, 845 (2000).
[11] L. A. Adamic, R. M. Lukose, A. R. Puniyani and B. A. Huberman, Phys. Rev. E 64, 041235 (2001).

[12] B. J. Kim, C. N. Yoon, S. K. Han and H. Jeong, Phys. Rev. E 65, 027103 (2002).

[13] H. Zhu and Z-X. Huan, Phys. Rev. E 70036117 (2004).

[14] A. P. S. de Moura, A. E. Motter and C. Grebogi, Phys. Rev. E 68036106 (2003).

[15] D. J. Watts, P. S. Dodds and M. E. J. Newman, Science 296, 1302 (2002).

[16] S. Carmi, R. Cohen and D. Dolev, Europhys. Lett. 74, 1102 (2006).

[17] H. P. Thadakamalla, R. Albert and S. R. T. Kumara, Phys. Rev. E 72, 066128 (2005).

[18] A. Clauset and C. Moore, preprint arxiv:cond-mat/0309415 198701 (2001).

[19] P. Sen, J. Stat. Mech. P04007 (2007).

[20] K. Basu Hajra and P. Sen, J. Stat. Mech. P06015 (2007).

[21] S. H. Lee, P-J. Kim, Y-Y. Ahn and H. Jeong, arXiv:0710.3268v1. 